\title{
Numerical solution for an almost square crack
}

\begin{abstract}
This paper studied the behaviour of the solution for an almost square crack, $\mathrm{q}$, in the plane elasticity. The problem of sliding the resulting shear forces can be formulated as a hypersingular integral equation over a considered domain, $\mathbf{q}$. The sharp corner of the square is rounded up such that the stress singularity is kept uniform form along the entire crack contour. The equation is then transformed into a similar hypersingular integral equation over a circular disc, D, using conformal mapping. The transformed hypersingular integral equation is afterward reduced to a system of linear algebraic equations using Galerkin method. The system of linear equations is solved numerically for the unknown coefficients, which later will be used in determining the stress intensity factors, maximum stress intensity and energy release rate. Comparison with the existing asymptotic solutions show a good agreement.
\end{abstract}

Keyword: Hypersingular boundary integral equation; Conformal mapping; Stress intensity factors; Energy release rate; Galerkin method 